\title{
Bipolar affective disorder, stress fractures, fungal dermopathy and 'tree frog fingers'
}

\author{
M Reuber, K Misch, V Patel
}

A 51-year-old woman was referred to the Department of Endocrinology following her visit to a dermatological out-patient clinic. She gave an eight-year history of medical problems: following the death of her father she had consulted her general practitioner with weight loss, anxiety, psychomotor retardation and suicidal ideation. After these symptoms had failed to improve on treatment with a tricyclic antidepressant she was admitted to the local psychiatric hospital. There she received eight courses of electroconvulsive therapy and was discharged on prophylactic lithium and tricyclic antidepressant therapy. Three years later the (premenopausal) patient suffered a stress fracture of her second left metatarsal, followed by stress fractures of her third and fourth left metatarsals one year later. A year later she noticed a puffy swelling of the pulp of her right thumb. Over the following two years, the left thumb, right ring and index fingers were all affected by similar changes and she became aware of a tendency to bruise easily.

In the year preceeding her presentation, the patient suffered a further stress fracture (third right metatarsal) and a recurrence of psychiatric symptoms four weeks after she had stopped her lithium and clomipramine therapy. Her sleep requirement dropped to three hours, her attention was poor and she became emotionally labile. Her maniacal symptoms precipitated a further psychiatric admission and the commencement of carbamazepine therapy. After her discharge, the patient's long-standing tinea pedis became so troublesome that she consulted a dermatologist.

Examination revealed no markedly plethoric facies, centripetal obesity or hirsutes. Pale striae were noted in both flanks. Body Mass Index was 28.3. There was extensive tinea pedis. The hand changes are illustrated in the figures. Skin thickness was reduced to less than $2 \mathrm{~mm}$. Her pulse was 72 beats/ min, blood pressure $170 / 110 \mathrm{mmHg}$. Otherwise cardiovascular and chest and abdominal examination were unremarkable. Neurological examination revealed no abnormalities. The dermatologist advised topical treatment of the intertriginous fungal infections. He was, however, intrigued by the frog-finger-like appearance of both her thumbs, her right ring and index fingers and suggested some simple screening tests, following which the patient was referred for endocrinological investigation.

Department of Endocrinology, St Bartholomew's Hospital, The Royal Hospitals NHS Trust, West Smithfield, London EC1A 7BE, UK $M$ Reuber

V Patel

Department of Dermatology, Epsom General Hospital NHS Trust, Epsom, Surrey KT18 7EG, UK K Misch

Correspondence to $\mathrm{Dr} M$ Reuber, MD MRCP, Department of

Endocrinology, Northern General Hospital, University of Sheffield, Herries Road, Sheffield, UK

Accepted 9 July 1997

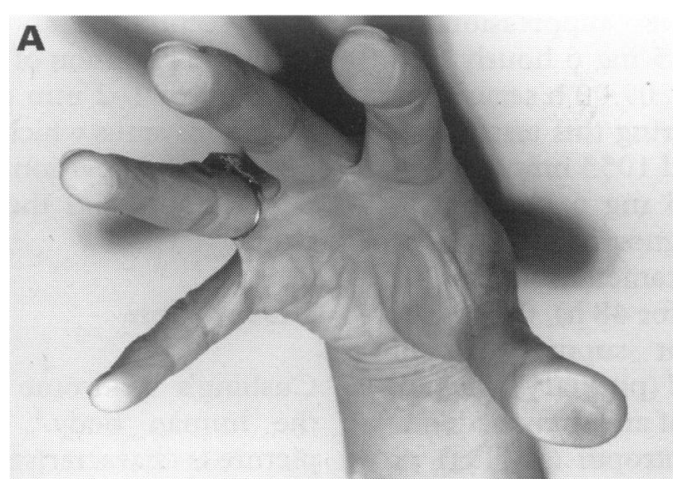

Figure 1 Right hand held loosely against glass pane
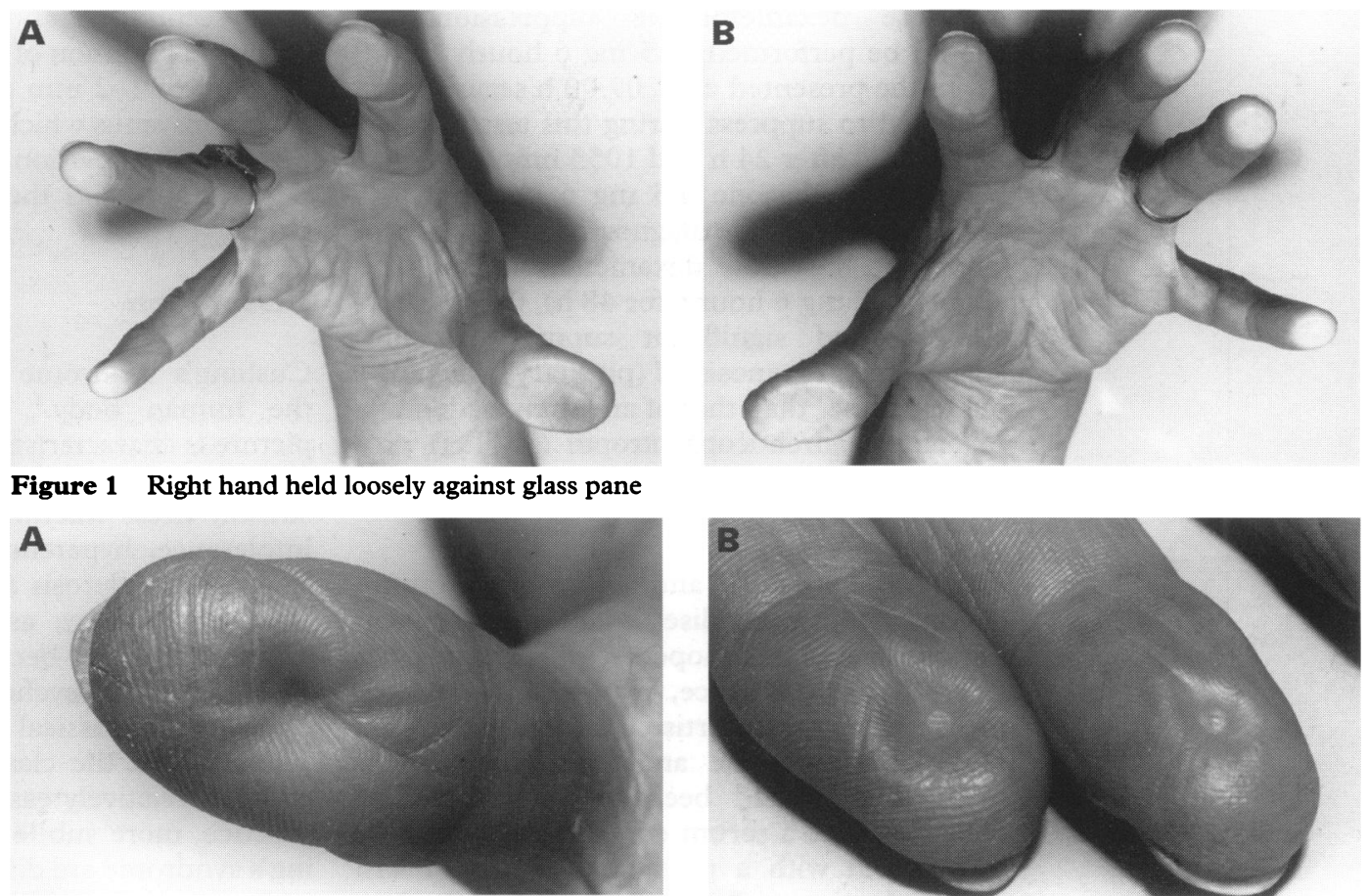

Figure 2 Reduced elasticity of the pulp of the affected terminal phalanges demonstrated by lack of restitution of the finger pulp $60 \mathrm{~s}$ after application of pressure with a round wooden stick (diameter $2 \mathrm{~mm}$ )

\section{Questions}

1 What diagnosis did the dermatologist suspect?

2 Which screening tests were performed to support the diagnosis?

3 Which further investigations were required before treatment could be undertaken? 


\section{Answers}

QUESTION 1

Cushing's syndrome is the only unifying diagnosis in this patient presenting with psychopathy, osteoporosis, dermopathy (easy bruising and skin fragility, reduced skin thickness, tinea pedis and tree frog fingers ${ }^{1}$ ), mild obesity and hypertension. Like hypercortisolism, thyrotoxicosis may initially present with pychiatric symptoms, and it is a cause of osteoporosis. It may also present as a dermopathy (pretibial myxoedema, infiltrative ophthalmopathy) and is associated with distal finger changes (thyroid acropatchy). The digital changes, however, resemble clubbing and do not affect the pulp of the finger tip.

QUESTION 2

Unfortunately a random cortisol estimation is not a very useful diagnostic test in this setting. The initial screening test performed was a 24-h urine free cortisol (UFC). This was found to be raised at $640 \mathrm{nmol} / 24 \mathrm{~h}$ (reference range $25-280 \mathrm{nmol} / 24 \mathrm{~h}$ ). Whilst measurement of UFC is a good screening procedure (elevated in $90 \%$ of patients with Cushing's syndrome) alternatives would have included a cortisol day curve (midnight cortisol normally less than $50 \%$ of $09.00 \mathrm{~h}$ value) and a $1 \mathrm{mg}$ overnight dexamethasone suppression test. All of these tests have their drawbacks, particularly in the setting of psychiatric illness, stress, drug treatment, hormone replacement therapy and oral contraception, obesity and alcoholism. If any of the screening tests are abnormal or borderline and there is a high clinical index of suspicion, a low-dose dexamethasone suppression test should be performed $(0.5 \mathrm{mg} 6$ hourly for 48 h). In the presented case, $09.00 \mathrm{~h}$ serum cortisol failed to suppress during this test (cortisol $825 \mathrm{nmol} / 1$ after $24 \mathrm{~h}$ and $1055 \mathrm{nmol} / 1$ after 48 $\mathrm{h}$ of dexamethasone $0.5 \mathrm{mg} 6$ hourly). This result established the diagnosis of hypercortisolism. In a high-dose dexamethasone suppression test ( $2 \mathrm{mg} 6$ hourly for $48 \mathrm{~h}$ ), $09.00 \mathrm{~h}$ cortisol showed significant suppression, thus suggesting a diagnosis of (pituitary) Cushing's disease rather than that of an adrenal adenoma or ectopic adrenocorticotropin (ACTH) production.

QUESTION 3

After haematological and biochemical complications of Cushing's disease had been excluded (leucocytosis, lymphopenia, thrombocytosis, electrolyte disturbance, hyperglycaemia), the presence of hypercortisolism was confirmed once carbamazepine and hormone replacement therapy had been discontinued. The mean value of a serum cortisol day curve was $457 \mathrm{nmol} / \mathrm{l}$ with a midnight cortisol of 413 $\mathrm{nmol} / \mathrm{l}$ (patient asleep). Low and high dose dexamethasone suppression corroborated the outcome of the earlier tests. Further investigations were aimed at identifying the cause of the patient's hypercortisolaemia. Biochemical tests included a corticotropin-releasing factor (CRF) test, which revealed a blunted ACTH and cortisol response with values rising from
23 to $39 \mathrm{ng} / \mathrm{l}$ and 474 to $678 \mathrm{nmol} / \mathrm{l}$, respectively. This supported a diagnosis of (pituitary) Cushing's disease. Bilateral inferior petrosal sinus catheter sampling gave no indication of the site of a possible ACTHproducing pituitary adenoma (ACTH rise after CRF stimulation on the right from 22 to 85 $\mathrm{ng} / \mathrm{l}$, on the left from 22 to $98 \mathrm{ng} / \mathrm{l}$ ). Radiological investigations included normal chest and skull radiographs. Computed tomography of the pituitary showed a bulky gland filling the fossa, with a slightly convex upper surface. Magnetic resonance imaging showed a pituitary fossa floor sloping downwards anteriolaterally to the right side with a suggestion of a low signal lesion in the right pituitary lobe. A slightly bulky gland seemed to deviate the pituitary stalk to the left. In view of the history of stress fractures DXA-densitometry was performed, revealing bone density to be $67-79 \%$ below the mean of females of the patient's age.

A diagnosis was made of Cushing's disease caused by a pituitary microadenoma, possibly on the right. The patient was referred for transsphenoidal hypophysectomy. The immunhistochemical examination of the excised specimen revealed a chromophobe adenoma with positive immunostaining for ACTH and negative staining for other pituitary hormones. Some areas in the anterior pituitary showed Crookes' hyaline change. This is an ultrastructural feature associated with ACTH-producing pituitary adenomas, consisting of bundles of fine perinuclear filaments.

Despite the histological proof of successful surgery, postoperative $09.00 \mathrm{~h}$ cortisol remained detectable (472 ng/1 six days post-op), and a second operation was thought to be necessary. Exploration of the sella revealed a tissue segment of 1-2 mm diameter adherent to the cavernous sinus which was duely excised. After this surgical revision, cortisol values became undetectable and the patient was considered cured.

\section{Discusssion}

Cushing's syndrome affects most systems of the human body. ${ }^{2}$ The full-blown clinical picture is characterised by progressive specificsite adiposity, myopathy, dermopathy, hirsutism, stress fractures, psychopathy, glucose intolerance, hypercholesterolaemia, hypertension, atherosclerosis and immunosuppression. The psychopathy, as illustrated in the presented case, may become manifest as depression, mania or psychosis and can preceed the onset of the classical physical signs by several years. Whilst the classical syndrome outlined above is relatively easy to recognise in clinical practice, more subtle manifestations of Cushing's syndrome are difficult to spot and characterise, particularly in the setting of concomitant medical conditions such as obesity, alcoholism and depression. In the case presented here, the diagnosis was suspected in a dermatological out-patient department on the basis of a recently described sign.

Dermatological complications of persistent hypercortisolism include fungal infections 
whose spread and persistence are thought to be caused by cortisol-mediated reduction of IL-1 secretion from monocytes/macrophages and IL-2 release from activated lymphocytes resulting in a suppression of T-lymphocyte proliferation. The most characteristic skin changes of Cushing's syndrome, however, are the result of an abnormal collagen metabolism. Easy bruising and violaceous striae formation are considered to be due to reduced collagen-platelet aggregation and cortisol-induced protein catabolism in blood vessel walls. Collagen catabolism and increased turnover also cause abnormalities of bone architecture leading to stress fractures. It has recently been shown that various serum parameters of collagen turnover such as type I procollagen and aminoterminal propeptide of type III procollagen are increased in Cushing's syndrome. ${ }^{3}$

In the seminal report of profound acquired digital collagen atrophy (tree frog fingers), histological examination of the digital pulp showed focally decreased amounts of normal collagen with obvious dermal thinning. The dermal elastin fibres were noted to be of normal morphology but clumped as a consequence of the loss of collagen. These histological findings were broadly similar to more generalised cutaneous changes reported in previous studies of the effect of hypercortisolism on skin structure. ${ }^{4}$ As demonstrated in the digital photographs, the patient presented here exhibited a profound reduction of cutaneous elasticity in her affected fingers.

The case reported by Groves et al described similar changes in a patient with Cushing's syndrome due to a benign adrenal adenoma. The patient described here represents the first case of marked digital cutis laxa due to Cushing's syndrome caused by a pituitary adenoma (Cushing's disease). Our report high-

1 Groves RW, MacDonald LM, MacDonald D M. Profound digital collagen atrophy: a new cutaneous presentation of adrenal-dependent Cushing's syndrome. Br $f$ Dermatol 1990;123:667-71

2 Yanovski JA, Cutler GB Jr. Glucocorticoid action and the Yanovski JA, Cutler GB Jr. Glucocorticoid action and the clinical features of Cushing's synd

3 Piovesan A, Terzolo M, Reimondo G, et al. Biochemical markers of bone and collagen turnover in acromegaly or Cushing's syndrome. Horm Metab Res 1994;26:234-7.

\section{Learning points}

Cushing's syndrome: the atypical presentation

- depression, mania, psychosis

- osteoporosis and stress fractures

- opportunistic infections, osteomyelitis

- dermopathy

- diabetes

- hypertension

- glaucoma, cataract

- amenorrhoea

Dermopathy of hypercortisolism

- easy bruising

- skin thickness $<2 \mathrm{~mm}$

- violaceous striae

- increased fragility and poor healing

- tinea and candidal infection

- digital cutis laxa (tree frog fingers)

lights the diagnostic challenge that Cushing's syndrome continues to present. Dermatological changes serve to discriminate between true hypercortisolism and differential diagnoses including obesity, polycystic ovary syndrome and depression. Like skin-fold thickness, ${ }^{5}$ profound digital collagen atrophy with reduced elasticity should be looked for as a sign of Cushing's syndrome.

\section{Final diagnosis}

Cushing's disease caused by a pituitary microadenoma.

Keywords: Cushing's disease; dermopathy; collagen

We are greatly indebted to Prof JAH Wass for allowing us to report this patient's clinical mangagement at the Department of Endocrinology, St Bartholomew's Hospital, London. The patient herself is acknowledged for her cooperation and her permission to publish this report.

4 Gabrilove JL, Schwartz A, Churg J. Effect of hormones on the skin in endocrinological disorders. $f$ Clin Endocrinol 1962;22:688-92.

5 Corenblum B, Kwan T, Gee S, Wong NC. Bedside assessment of skin-fold thickness. A useful measurement for distinguishing Cushing's disease from other causes of hirsutism and oligomenorrhoea. Arch Intern Med 1994;154: $777-81$. 